\title{
Outcomes of pelvic exenteration for recurrent or primary locally advanced colorectal cancer
}

\author{
Hwa Yeon Yang, Sung Chan Park, Jong Hee Hyun, Ho Kyung Seo', Jae Hwan Oh \\ Center for Colorectal Cancer, ${ }^{1}$ Center for Prostate Cancer, Research Institute and Hospital, National Cancer Center, Goyang, Korea
}

Purpose: The objective of this study was to assess the clinical outcomes of pelvic exenteration for patients with primary locally advanced colorectal cancer (LACRC) or locally recurrent colorectal cancer (LRCRC), and to identify clinically relevant prognostic factors.

Methods: Between January 2001 and December 2010, 40 consecutive patients with primary LACRC or LRCRC underwent pelvic exenteration at the National Cancer Center, Republic of Korea. We retrospectively reviewed their medical records. Results: The median age was 59 years and the median follow-up time was 26 months (range, 1-117 months). The overall complication and in-hospital mortality rates were $70 \%(28 / 40)$ and $7.5 \%(3 / 40)$, respectively. The complication rates were similar between patients with primary LACRC $(69.6 \%)$ and those with LRCRC $(70.6 \%)$. The overall recurrence rate was $50 \%$ (17/34), and was lower in patients with primary LACRC than in patients with LRCRC (33.3\% vs. 76.9\%, P = 0.032). The 5-year overall survival was significantly different between primary LACRC and patients with LRCRC (58.7\% vs. $11.8 \%, P=0.022)$. Multivariate analysis revealed that radicality (R0 vs. R1/R2) was an independent prognostic factor for overall survival $(P=$ 0.020).

Conclusion: The complication and operative mortality rates of pelvic exenteration remained high, but pelvic exenteration might provide an opportunity for long-term survival and good local control. Complete (RO) resection was the only independent prognostic factor for overall survival.

[Ann Surg Treat Res 2015;89(3):131-137]

Key Words: Pelvic exenteration, Colorectal neoplasms, Local neoplasm recurrences

\section{INTRODUCTION}

Carcinoma of the colon and rectum can directly invade or adhere to other organs or tissue structures. Recent data suggest that approximately $10 \%$ of primary rectal carcinomas present with tumor invasion into adjacent organs without distant metastases [1,2]. The wider application of total mesorectal excision and multimodal treatment regimens, including neoadjuvant and adjuvant therapy, have reduced the rate of local recurrence to $<8 \%$ [3]. However, many patients with local recurrence exhibit disabling complications, including severe pain caused by bony or nervous tissue involvement, urinary obstruction, fecal obstruction or incontinence, or persistent bleeding. In patients with primary locally advanced colorectal cancer (LACRC) or locally recurrent colorectal cancer (LRCRC), en bloc excision of the tumor and/or adjacent organs, via pelvic exenteration, is often necessary to obtain a negative surgical margin [4].

Total pelvic exenteration implies en bloc resection of the rectum, distal colon, bladder, lower ureter, internal reproductive organs, draining lymph nodes, and pelvic peritoneum. The procedure was first described by Brunschwig [5] in
Received February 5, 2015, Revised April 13, 2015,

Accepted April 24, 2015

Corresponding Author: Jae Hwan Oh

Center for Colorectal Cancer, National Cancer Center, 323 IIsan-ro, Ilsandong-gu, Goyang 410-769, Korea

Tel: +82-31-920-1535, Fax: +82-31-920-1148

E-mail: jayoh@ncc.re.kr
Copyright (c) 2015, the Korean Surgical Society

(c) Annals of Surgical Treatment and Research is an Open Access Journal. All articles are distributed under the terms of the Creative Commons Attribution NonCommercial License (http://creativecommons.org/licenses/by-nc/4.0/) which permits unrestricted non-commercial use, distribution, and reproduction in any medium, provided the original work is properly cited. 
1948 as a palliative operation for advanced cervical cancer. Pelvic exenteration for primary rectal cancer can provide a meaningful extension in long-term survival, but the relevance of pelvic exenteration for the treatment of recurrent cancer is still debated. Moreover, this operation is associated with high morbidity and mortality $[2,6]$.

Therefore, the primary objective of this study was to assess the clinical outcomes of pelvic exenteration with an ileal conduit for patients with primary LACRC or LRCRC. Our secondary objective was to identify clinically relevant prognostic factors for patient outcomes.

\section{METHODS}

Between January 2001 and December 2010, 40 consecutive patients with primary LACRC or LRCRC underwent pelvic exenteration at the National Cancer Center, Republic of Korea. We retrospectively reviewed their medical records. Preoperative diagnostic tests included physical examination, colonoscopy, endorectal ultrasound for patients with rectal cancer, measurement of the serum CEA level, contrast-enhanced CT scans of the chest and abdomen to evaluate distant disease, and CT or MRI to assess resectability status. PET-CT was also performed in selected patients. All of the patients were then discussed in a multidisciplinary team conference for the purpose of determining optimal treatment. In our institute, pelvic exenteration is performed in most all surgically tolerant patients with locally advanced colorectal carcinoma which directly invade or adhere to other organs or tissue structures. However, when surgical margin is threatened, neoadjuvant chemoradiotherapy is considered in multidisplinary team conferences according to tumor location and previous history of radiotherapy.

All patients underwent pelvic exenteration, which included total cystectomy and ileal conduit (Bricker) diversion. Total pelvic exenteration was defined as en bloc removal of the rectum, anus, urinary bladder, lower ureters, and internal reproductive organs. In modified pelvic exenteration, the lower rectum or anus was retained in situ or intestinal continuity was reestablished after removing the upper rectum, internal reproductive organs, urinary bladder, and lower ureters. The operation was performed by a multidisciplinary team, which included a colorectal surgeon, an urologist, a gynecologist, a vascular surgeon, an orthopedic surgeon, and a plastic surgeon.

The study was approved by the Institutional Review Board at the National Cancer Center (NCCNCS-12-600), Republic of Korea. The clinical parameters analyzed included patient characteristics, operative outcomes, pathologic outcomes, complications, and follow-up data.

All of the patients attended our clinic for standardized follow ups every 3 or 6 months. At each visit, we conducted a physical examination, digital rectal examination, complete blood count, liver function tests, and measured the serum CEA level. CT scans of the chest, abdomen, and pelvis, with or without PET-CT, were performed every 6 months. Recurrence was pathologically confirmed by direct biopsy or cytology, and/ or radiology. Local recurrence was defined as disease recurrence within the pelvic cavity. Distant metastasis was defined as recurrence outside the pelvic cavity.

\section{Statistical analysis}

The chi-square test, Fisher exact test, and t-tests were used to compare the clinical parameters between each group. Survival time was calculated as the time from the date of surgery to June 31,2012 , or death of any cause. Kaplan-Meier curves were used to assess overall survival. Univariate and multivariate analyses of factors associated with survival comparison were performed using log-rank tests and Cox proportional hazards model, respectively. Values of $\mathrm{P}<0.05$ were considered to indicate statistical significance.

\section{RESULTS}

The characteristics of the 40 patients who underwent pelvic exenteration are summarized in Table 1 . Their median age was 59 years (range, 38-84 years). There were 32 men (80\%) and 8 women (20\%). The median follow-up time was 26 months (range, 1-117 months). The primary tumor sites were the rectum ( $\mathrm{n}=$

Table 1. Baseline characteristics of the patients $(n=40)$

\begin{tabular}{lc}
\hline \multicolumn{1}{c}{ Characteristic } & Value \\
\hline Age (yr), median (range) & $59(38-84)$ \\
Sex & $32(80.0)$ \\
Male & $8(20.0)$ \\
Female & \\
Primary site & $28(70.0)$ \\
Rectum & $12(30.0)$ \\
Sigmoid & \\
Tumor classification & $23(57.5)$ \\
Primary LACRC & $17(42.5)$ \\
LRCRC & $19(47.5)$ \\
Type of operation & $21(52.5)$ \\
Total PE & \\
Modified PE & $27(67.5)$ \\
Radicality & $7 / 6(17.5 / 15.0)$ \\
R0 & $8 / 4(20.0 / 10.0)$ \\
R1/R2 & $31(77.5)$ \\
Preoperative CRT/prior RT & \\
Adjuvant chemotherapy &
\end{tabular}

Values are presented as number (\%) unless otherwise indicated. LACRC, locally advanced colorectal cancer; LRCRC, locally recurrent colorectal cancer; PE, pelvic exenteration; CRT, chemoradiotherapy; RT, radiotherapy. 
28) and sigmoid colon $(n=12)$. Pelvic exenteration was done for primary tumors in 23 patients and for recurrent tumors in 17 patients. Total pelvic exenteration was performed in 19 patients and modified pelvic exenteration was performed in 21 patients. R0 resection was achieved in 27 patients $(67.5 \%)$, and there were 7 R1 and 6 R2 resections. Partial sacrectomy was performed in one patient for recurrent rectal cancer. Twelve patients (30\%) received a fluoropyrimidine-based chemoradiotherapy regimen preoperatively or prior to radiotherapy for recurrent patients. Radiotherapy generally consisted of a total dose of $50.4 \mathrm{~Gy}$, which included $45 \mathrm{~Gy}$ in 25 fractions administered to the pelvis and a 5.4 Gy boost that was administered in three fractions to the primary tumor over 5.5 weeks. Thirty-one patients (77.5\%) received postoperative chemotherapy, which consisted of a fluoropyrimidine regimen, or an irinotecan- or oxaliplatinbased regimen.

Most patients with primary LACRC $(16 / 23,69.6 \%)$ underwent modified pelvic exenteration, while most patients with LRCRC (12/17, 70.6\%) underwent total pelvic exenteration. This difference in the rates of modified pelvic exenteration and total pelvic exenteration between these two groups of patients was statistically significant $(P=0.012)$. The mean hospital stay for patients with primary LACRC and LRCRC was 29 days and 51 days, respectively. The mean hospital stay and the duration of surgery were significantly shorter for patients with primary LACRC than for patients with LRCRC. The mean volume of transfused packed red blood cells was 3.8 units and 8.3 units in primary LACRC and LRCRC, respectively. R0 resection was

Table 2. Operative results in 40 patients with PE

\begin{tabular}{lccc}
\hline \multicolumn{1}{c}{ Variable } & $\begin{array}{c}\text { Primary } \\
\text { LACRC }\end{array}$ & LRCRC & P-value \\
& & & 0.041 \\
Primary site & $13(56.5)$ & $15(88.2)$ & \\
$\quad$ Rectum & $10(43.5)$ & $2(11.8)$ & \\
$\quad$ Sigmoid & & & 0.015 \\
CEA level (ng/dL) & $6(26.1)$ & $11(64.7)$ & \\
$\quad<5$ & $17(73.9)$ & $6(35.3)$ & \\
$\geq 5$ & & & 0.012 \\
Type of operation & $7(30.4)$ & $12(70.6)$ & \\
$\quad$ Total PE & $16(69.6)$ & $5(29.4)$ & \\
$\quad$ Modified PE & $19(82.7)$ & $8(47.1)$ & \\
Radicality & $4(17.3)$ & $9(52.9)$ & \\
$\quad$ R0 & $481.0 \pm 87.0$ & $641.7 \pm 163.3$ & 0.001 \\
$\quad$ R1/R2 & $29.2 \pm 18.0$ & $51.4 \pm 29.0$ & 0.010 \\
Duration of surgery (min) & $3.8 \pm 3.4$ & $8.3 \pm 10.1$ & 0.078 \\
Hospital stay (day) & & \\
PRBC volume (unit) & & & \\
\hline
\end{tabular}

Values are presented as number $(\%)$ or mean \pm standard deviation.

$\mathrm{PE}$, pelvic exenteration; LACRC, locally advanced colorectal cancer; LRCRC, locally recurrent colorectal cancer; PRBC, packed red blood cells. achieved in 19 of 23 patients (82.6\%) with primary LACRC and in 8 of 17 patients (47.1\%) with LRCRC, which was significantly different $(\mathrm{P}=0.038)$ (Table 2).

The tumor-node-metastasis (TNM) stage of the resected primary LACRCs and Wanebo classification of the LRCRCs are summarized in Table 3. In three patients with LRCRC, there were no lymph nodes found in the pathological specimens. Inflammatory adhesion rather than tumor invasion into adjacent organs was observed in 3 of 23 patients with primary LACRC and in 1 of 17 patients with LRCRC who were suspected

Table 3. Pathologic outcomes

\begin{tabular}{lrrr}
\hline \multicolumn{1}{c}{ Variable } & $\begin{array}{c}\text { Primary } \\
\text { LACRC }\end{array}$ & LRCRC & P-value \\
\hline Tumor differentiation & & & 0.631 \\
WD or MD & $21(91.3)$ & $13(81.3)$ & \\
$\quad$ PD or mucinous & $2(8.7)$ & $3(18.8)$ & \\
Tumor status & & & 0.624 \\
pT4 & $20(87.0)$ & $16(94.1)$ & \\
pT3 & $3(13.0)$ & $1(5.9)$ & \\
Node status & & & 0.896 \\
$\quad$ Negative & $9(39.1)$ & $7(41.2)$ & \\
$\quad$ Positive & $14(60.9)$ & $10(58.8)$ & \\
Angiolymphatic invasion & & & 0.371 \\
$\quad$ Absent & $5(21.7)$ & $1(6.7)$ & \\
$\quad$ Present & $18(78.3)$ & $14(93.3)$ & \\
Venous invasion & & & 0.101 \\
$\quad$ Absent & $11(47.8)$ & $3(20.0)$ & \\
$\quad$ Present & $12(52.2)$ & $12(80.0)$ & \\
Perineural invasion & & & 0.944 \\
$\quad$ Absent & $11(47.8)$ & $7(46.7)$ & \\
$\quad$ Present & $12(52.2)$ & $8(53.3)$ & \\
\hline
\end{tabular}

Values are presented as number (\%).

LACRC, locally advanced colorectal cancer; LRCRC, locally recurrent colorectal cancer; WD, well differentiated; MD, moderately differentiated; PD, poorly differentiated.

Table 4. Complications after pelvic exenteration

\begin{tabular}{lc}
\hline \multicolumn{1}{c}{ Complication } & No. $(\%)$ \\
Wound problem & $6(15)$ \\
Perineal wound dehiscence & $1(2.5)$ \\
Pelvic abscess & $10(25)$ \\
Anastomotic leakage & $2(5)$ \\
Bowel obstruction (con/op) & $3 / 3(7.5 / 7.5)$ \\
Urinary tract infection & $2(5)$ \\
Hydronephrosis & $2(5)$ \\
Pneumonia & $3(7.5)$ \\
Postoperative bleeding & $1(2.5)$ \\
Enterocutaneous fistula & $1(2.5)$ \\
Stoma necrosis & $1(2.5)$ \\
\hline
\end{tabular}

Values are presented as number (\%). con, conservative; op, operation. 
Table 5. Recurrence patterns in patients with R0/R1 resection $(n=34)$

\begin{tabular}{|c|c|c|c|c|}
\hline Recurrenc type & No. & Primary LACRC $(n=21)$ & $\operatorname{LRCRC}(n=13)$ & P-value \\
\hline Patients with recurrence & 17 & $7(33.3)$ & $10(76.9)$ & 0.032 \\
\hline Local alone & 3 & $1(4.3)$ & $2(11.8)$ & \\
\hline Local and distant & 7 & $2(8.7)$ & $5(29.4)$ & \\
\hline Distant alone & 7 & $4(17.4)$ & $3(17.6)$ & \\
\hline Local recurrence & 10 & $3(14.3)$ & $7(53.8)$ & 0.022 \\
\hline
\end{tabular}

Values are presented as number (\%).

Six patients with $\mathrm{R} 2$ resection were excluded from this analysis.

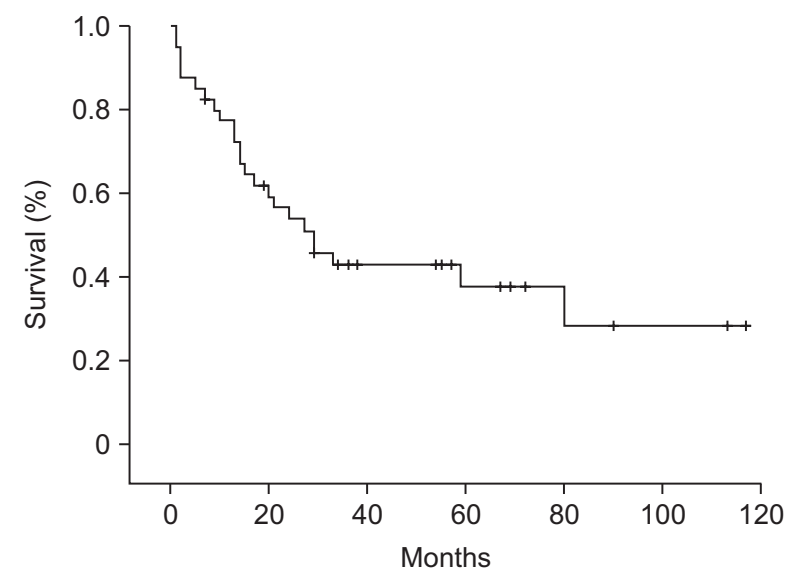

Fig. 1. Kapla-Meier analysis of overall survival after pelvic exenteration in patients with primary advanced or recurrent colorectal cancer.

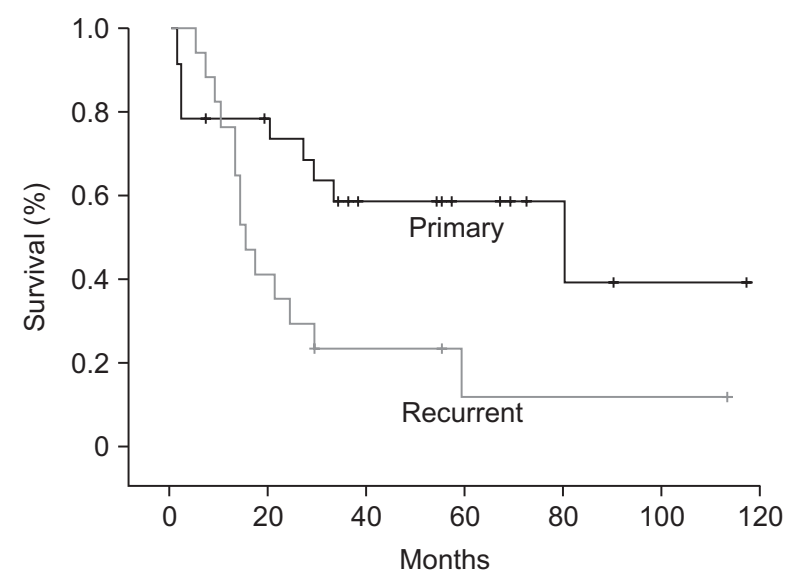

Fig. 2. Comparison of overall survival after pelvic exenteration between patients with primary locally advanced colorectal carcinoma and patients with locally recurrent colorectal cancer (Kaplan-Meier analysis; log-rank test, $\mathrm{P}=$ 0.022).

of having T4 involvement at preoperative or intraoperative evaluation. There were no significant differences in tumor differentiation, $\mathrm{T}$ status, $\mathrm{N}$ status, angiolymphatic invasion, venous invasion, or perineural invasion between the two

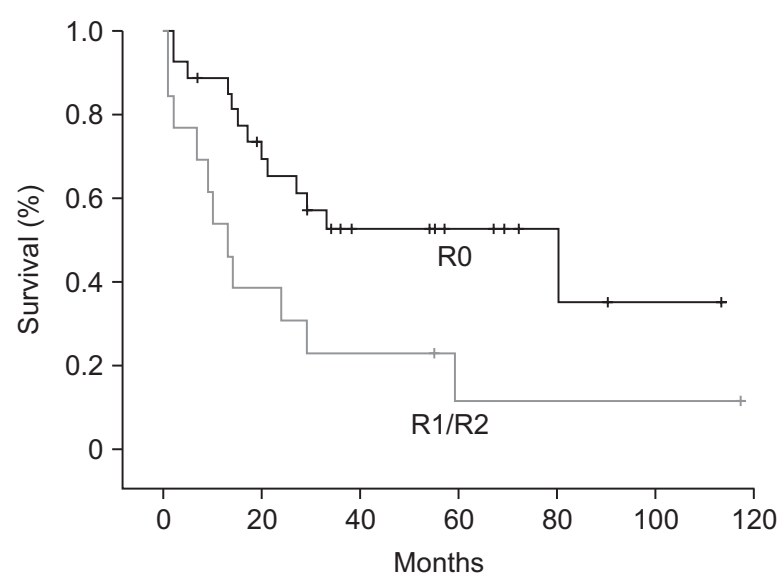

Fig. 3. Comparison of overall survival after complete (R0) and incomplete (R1 : R2) total pelvic exenteration (Kaplan-Meier analysis; log-rank test, $\mathrm{P}=0.015$ ).

groups.

The postoperative complication profiles are described in Table 4. The overall complication rate was 70\% (28/40) and 30\% (12/40) required additional surgery, including 5 patients who underwent pelvic abscess irrigation and debridement under spinal anesthesia. The rates of complications were similar between patients with primary LACRC $(69.6 \%)$ or LRCRC (70.6\%). The most common complications were pelvic abscess (25\%), followed by wound problems (7.5\%), and postoperative bowel obstruction (7.5\%). Of 6 patients with bowel obstruction, 3 underwent an operative intervention and three were managed conservatively with Levin-tube drainage. There was no death of patients during the first 30 days after surgery. However, 3 patients $(7.5 \%)$ with primary LACRC died during the postoperative hospital stay period.

The median follow-up time for all patients was 25 months (range, 1-117 months). Except for 6 patients with R2 resection, the overall recurrence rate was $50 \%(17 / 34)$ and the rate was lower in patients with primary LACRC than in patients with LRCRC (33.3\% vs. 76.9\%, $\mathrm{P}=0.032$ ). The local recurrence rate was also lower in patients with primary LACRC than in patients with LRCRC (14.3\% vs. 53.8\%, $\mathrm{P}=0.022)$. Only 3 patients (8.8\%) had local recurrence alone (Table 5). The estimated overall 
Table 6. Univariate and multivariate analysis of factors associated with overall survival

\begin{tabular}{|c|c|c|c|c|c|c|}
\hline \multirow{2}{*}{ Variable } & \multirow{2}{*}{ No. } & \multicolumn{2}{|c|}{ Univariate analysis } & \multicolumn{3}{|c|}{ Multivariate analysis } \\
\hline & & 5-year OS (\%) & P-value & $\mathrm{HR}$ & $95 \% \mathrm{Cl}$ & P-value \\
\hline \multicolumn{7}{|l|}{ Age $(y r)$} \\
\hline$<60$ & 21 & 40.2 & & & & \\
\hline$\geq 60$ & 19 & 31.6 & 0.149 & & & \\
\hline \multicolumn{7}{|l|}{ Sex } \\
\hline Male & 32 & 45.3 & & & & \\
\hline Female & 8 & 16.7 & 0.307 & & & \\
\hline \multicolumn{7}{|l|}{ Primary site } \\
\hline Rectum & 28 & 32.5 & & & & \\
\hline Sigmoid & 12 & 48.6 & 0.613 & & & \\
\hline \multicolumn{7}{|l|}{ CEA level $(\mathrm{ng} / \mathrm{mL})$} \\
\hline$<5$ & 17 & 27.5 & & & & \\
\hline$\geq 5$ & 23 & 44.9 & 0.797 & & & \\
\hline \multicolumn{7}{|l|}{ Tumor type } \\
\hline Primary LACRC & 23 & 58.7 & & & & \\
\hline LRCRC & 17 & 11.8 & 0.022 & & & \\
\hline \multicolumn{7}{|l|}{ Type of operation } \\
\hline Total PE & 19 & 27.6 & & & & \\
\hline Modified PE & 21 & 49.0 & 0.370 & & & \\
\hline Radicality & & & & 2.613 & $1.165-5.860$ & 0.020 \\
\hline Ro & 27 & 52.7 & & & & \\
\hline R1/ R2 & 13 & 11.5 & 0.015 & & & \\
\hline
\end{tabular}

OS, overall survival; $\mathrm{HR}$, hazard ratio; $\mathrm{Cl}$, confidence interval; LACRC, locally advanced colorectal cancer; LRCRC, locally recurrent colorectal cancer; PE, pelvic exenteration.

survival of the 40 patients was $42.9 \%$ at 3 years and $28.1 \%$ at 5 years (Fig. 1). A significant difference in 5-year overall survival was found between primary LACRC and LRCRC $(58.7 \%$ vs. $11.8 \%, \mathrm{P}=0.022$ ) (Fig. 2). There was also statistically significant difference in 5-year overall survival between patients with $\mathrm{R} 0$ or $\mathrm{R} 1 / \mathrm{R} 2$ resection $(52.7 \%$ vs. $11.5 \%, \mathrm{P}=0015)$ (Fig. 3). The median survival time for patients who underwent pelvic exenteration for primary LACRC was 80 months versus 15 months in patients with LRCRC. Univariate analysis showed that tumor type (primary LACRC vs. LRCRC) and radicality (R0 vs. R1/R2) were significant prognostic factors for overall survival. However, in multivariate analysis, only radicality was an independent prognostic factor for overall survival $(\mathrm{P}=0.020$ ) (Table 6).

\section{DISCUSSION}

This study assessed whether pelvic exenteration was effective in patients with primary LACRC or LRCRC and identified useful prognostic factors for application in clinical settings. The 5-year survival rate in our study was $58.7 \%$ for patients with primary LACRC and 11.8\% for patients with LRCRC (Fig. 2). These outcomes are comparable with those reported in other studies [6-11]. The 5-year survival rate was significantly higher in patients who underwent complete ( $\mathrm{R} 0)$ pelvic exenteration than in patients who underwent incomplete (R1/R2) pelvic exenteration ( $P=0.015)$ (Fig. 3). Univariate analysis showed that tumor type (primary LACRC vs. LRCRC) and radicality (R0 vs. R1/R2) were significant prognostic factors for overall survival. However, in multivariate analysis, only radicality was an independent prognostic factor for overall survival $(\mathrm{P}=0.020)$ (Table 4). Therefore, one of the most important prognostic factors after pelvic exenteration is the status of the surgical margin, irrespective of the type of cancer.

It is not unusual for another organ to be involved in patients with a colorectal mass [2]. Therefore, at the time of surgery, it is often difficult to assess whether the involvement represents neoplastic invasion or inflammation [12]. Whenever possible, the involved organ should be resected en bloc. Pelvic exenteration allows en bloc excision of the tumor and/or adjacent organs, and provides a negative surgical margin. Pelvic exenteration for primary rectal cancer also has meaningful benefits in terms of extending long-term survival [13]. However, the prognosis after pelvic exenteration for recurrent rectal cancer is poorer [4]. Many studies have reported the outcomes of pelvic exenteration, but most case series are heterogenous in terms of the application of total cystectomy [7,9,12]. All of the patients in our study underwent colorectal resection with total cystectomy and ileal conduit diversion. Therefore, the present study provides more solid data on the outcomes of total cystectomy with ileal conduit diversion during CRC resection. 
In patients with LRCRC, the ability to perform complete surgical resection is the most important prognostic factor with respect to the clinical outcome. Tumor location and the degree of local invasion affect resectability. R0 resection can be accomplished in 30\%-60\% of patients with LRCRC [14]. In the present study, R0 resection was achieved in $47.1 \%$ of the patients with LRCRC, which was significantly lower than that in patients with primary LACRC (82.7\%). This lower rate of complete resection is accompanied by lower rates of local control and overall survival in patients with recurrent cancer $[10,15]$. In the present study, 7 of 13 patients $(53.8 \%)$ who had undergone R0/R1 resection for LRCRC experienced local recurrence, while the remaining 4 patients had $\mathrm{R} 2$ resection. This indicates a limitation of pelvic exenteration for recurrent tumors in terms of the local control rate. In our recent study, the 5-year locoregional relapse-free survival was $66.4 \%$ in patients who received chemoradiotherapy with an adjusted radiation dose [16]. Therefore, pelvic exenteration for LRCRC should be performed in properly selected patients.

The morbidity and mortality rates after pelvic exenteration were $70 \%$ and $7.5 \%$, respectively, which is within the reported ranges of $38 \%-70 \%$ and $0 \%-13 \%$, respectively $[6,7,12,15,17,18]$. The morbidity rate, particularly of complications requiring additional surgical procedures, was quite substantial in our patients. Additional surgical procedures were necessary in 12 patients (30\%); the procedures were pelvic abscess irrigation and debridement in 5 patients, small bowel resection and anastomosis in 3 patients, postoperative bleeding control and ureterointestinal anastomosis revision in 1 patient, ileostomy because of small bowel obstruction in 1 patient, stoma revision in 1 patient, and wound repair because of abdominal wound dehiscence in 1 patient. Three patients with primary LACRC died during the postoperative hospital stay period because of aspiration pneumonia (on postoperative day 42), pneumonia (postoperative day 64), and graft pseudoaneurysm rupture after external iliac artery resection (postoperative day 64) in one patient each. The latter two patients presented with perforated primary sigmoid colon cancer and a localized abscess, and their deaths might be attributed to a septic condition associated with the perforated cancer [19].

Our study suggests that the complication rates and operative mortality for pelvic exenteration remain high, but pelvic exenteration might provide an opportunity to extend longterm survival and good local control. R0 resection was the only independent prognostic factor for overall survival in this cohort of patients.

\section{CONFLICTS OF INTEREST}

No potential conflict of interest relevant to this article was reported.

\section{REFERENCES}

1. Winter DC, Walsh R, Lee G, Kiely D, O'Riordain MG, O'Sullivan GC. Local involvement of the urinary bladder in primary colorectal cancer: outcome with enbloc resection. Ann Surg Oncol 2007;14:6973.

2. Pawlik TM, Skibber JM, Rodriguez-Bigas MA. Pelvic exenteration for advanced pelvic malignancies. Ann Surg Oncol 2006;13:612-23.

3. Rahbari NN, Ulrich AB, Bruckner T, Munter M, Nickles A, Contin P, et al. Surgery for locally recurrent rectal cancer in the era of total mesorectal excision: is there still a chance for cure? Ann Surg 2011;253:522-33.

4. Wanebo HJ, Antoniuk P, Koness RJ, Levy A, Vezeridis M, Cohen SI, et al. Pelvic resection of recurrent rectal cancer: tech- nical considerations and outcomes. Dis Colon Rectum 1999:42:1438-48.

5. Brunschwig A. Complete excision of pelvic viscera for advanced carcinoma; a one-stage abdominoperineal operation with end colostomy and bilateral ureteral implantation into the colon above the colostomy. Cancer 1948;1:177-83.

6. Nielsen MB, Rasmussen PC, Lindegaard JC, Laurberg S. A 10-year experience of total pelvic exenteration for primary advanced and locally recurrent rectal cancer based on a prospective database. Colorectal Dis 2012;14:1076-83.

7. Gannon CJ, Zager JS, Chang GJ, Feig BW, Wood CG, Skibber JM, et al. Pelvic exenteration affords safe and durable treatment for locally advanced rectal carcinoma. Ann Surg Oncol 2007;14:1870-7.
8. Lopez-Basave HN, Morales-Vasquez F, Herrera-Gomez A, Rosciano AP, MenesesGarcia A, Ruiz-Molina JM. Pelvic exenteration for colorectal cancer: oncologic outcome in 59 patients at a single institution. Cancer Manag Res 2012;4:351-6.

9. Kakuda JT, Lamont JP, Chu DZ, Paz IB. The role of pelvic exenteration in the management of recurrent rectal cancer. Am J Surg 2003;186:660-4.

10. Vermaas M, Ferenschild FT, Verhoef C, Nuyttens JJ, Marinelli AW, Wiggers $\mathrm{T}$, et al. Total pelvic exenteration for primary locally advanced and locally recurrent rectal cancer. Eur J Surg Oncol 2007:33:452-8.

11. Domes TS, Colquhoun PH, Taylor B, Izawa JI, House AA, Luke PP, et al. Total pelvic exenteration for rectal cancer: outcomes 
and prognostic factors. Can J Surg 2011:54:387-93.

12. Ishiguro S, Akasu T, Fujita S, Yamamoto S, Kusters M, Moriya Y. Pelvic exenteration for clinical T4 rectal cancer: oncologic outcome in 93 patients at a single institution over a 30-year period. Surgery 2009;145:189-95.

13. Kang H, Kim HG, Ju JK, Kim DY. Multivisceral resection for locally advanced rectal cancer: adequate length of distal resection margin. J Korean Surg Soc 2012:82:87-93.

14. Bouchard P, Efron J. Management of recurrent rectal cancer. Ann Surg Oncol 2010;17:1343-56.

15. Yamada K, Ishizawa T, Niwa K, Chuman Y, Aikou T. Pelvic exenteration and sacral resection for locally advanced primary and recurrent rectal cancer. Dis Colon Rectum 2002;45:1078-84.

16. Lee JH, Kim DY, Kim SY, Park JW, Choi $\mathrm{HS}, \mathrm{Oh} \mathrm{JH}$, et al. Clinical outcomes of chemoradiotherapy for locally recurrent rectal cancer. Radiat Oncol 2011;6:51.

17. Ike H, Shimada H, Ohki S, Yamaguchi S, Ichikawa Y, Fujii S. Outcome of total pelvic exenteration for locally recurrent rectal cancer. Hepatogastroenterology 2003:50:700-3.

18. Nishio M, Sakakura C, Nagata T, Miyashita A, Hamada T, Ikoma H, et al. Outcomes of total pelvic exenteration for colorectal cancer. Hepatogastroenterology 2009:56:1637-41.

19. Abdelrazeq AS, Scott N, Thorn C, Verbeke CS, Ambrose NS, Botterill ID, et al. The impact of spontaneous tumour perforation on outcome following colon cancer surgery. Colorectal Dis 2008; 10:775-80. 